\title{
Atomic scale investigation of grain boundary structure role on the intergranular deformation in aluminum
}

\author{
I. Adlakha ${ }^{1}$, M.A. Bhatia ${ }^{1}$, M.A. Tschopp ${ }^{2}$, and K.N. Solanki ${ }^{1 *}$ \\ ${ }^{1}$ School for Engineering of Matter, Transport, and Energy; Arizona State University; Tempe, AZ \\ 85287, USA \\ ${ }^{2}$ Weapons and Materials Research Directorate, Aberdeen Proving Ground, MD 21005, USA \\ *(480)965-1869; (480)727-9321 (fax), E-mail: kiran.solanki@asu.edu, (Corresponding author)
}

\begin{abstract}
The role that grain boundary (GB) structure plays on the directional asymmetry of an intergranular crack (i.e. cleavage behavior is favored along one direction, while ductile behavior along the other direction of the interface) was investigated using atomistic simulations for aluminum $<110>$ symmetric tilt grain boundaries. Middle-tension $(\mathrm{M}(\mathrm{T}))$ and Mode-I crack propagation specimens were used to evaluate the predictive capability of the Rice criterion. The stress-strain response of the GBs for the $\mathrm{M}(\mathrm{T})$ specimens highlighted the importance of the GB structure. The observed crack tip behavior for certain GBs ( $\Sigma 9$ (221), $\Sigma 11$ (332) and $\Sigma 33$ (441) with the $\mathrm{M}(\mathrm{T})$ specimen displayed an absence of directional asymmetry which is in disagreement with the Rice criterion. Moreover, in these GBs with the $\mathrm{M}(\mathrm{T})$ specimen, the dislocation emission from a GB source at a finite distance ahead of the crack tip was observed rather than from the crack tip, as suggested by the Rice criterion. In an attempt to understand discrepancy between the theoretical predictions and atomistic observations, the effect of boundary conditions (M(T), Mode-I and the edge crack) on the crack tip events was examined and it was concluded that the incipient plastic events observed were strongly influenced by the boundary conditions (i.e. activation of dislocation sources along the GB, in contrast to dislocation nucleation directly from the crack tip). In summary, these findings provide new insights into crack growth behavior along GB interfaces and provide a physical basis for examining the role of the GB character on incipient event ahead of a crack tip and interface properties, as an input to higher scale models.
\end{abstract}

Keywords: Grain boundary; Dislocation; Fracture; Directional anisotropy 


\section{Introduction}

Understanding the mechanisms of fracture in materials is important for predicting failure in materials and, ultimately, for engineering new materials for enhanced fracture properties and/or longer life cycles in their intended applications. Experimental and fundamental studies are being explored to understand material failure at multiple length and time scales. Recently, several studies using atomistic methods have dedicated considerable effort towards understanding the brittle versus ductile behavior of materials, including the dynamic instabilities that occur during fracture [1-12]. In particular, some of these studies focus on the deformation process at the crack tip, i.e., brittle (cleavage) versus ductile (nucleation of partial and full dislocations, and deformation twinning) behavior and dislocation burst in single crystal and bicrystal metals. These simulations have clarified the effects of applied load orientation and slip planes on dislocation nucleation at/ahead of the crack tip along the grain boundaries (GBs). Hence, the role of the GB structure becomes pertinent in understanding the interfacial crack deformation behavior.

Experimentally, in $\mathrm{Cu}$ and $\mathrm{Fe}$ bicrystals, it has been shown that there exists a strong directional dependence on the fracture behavior along the interface [13-18]. The observed behavior was consistent with the Rice-Thompson (RT) criterion [19] prediction for the dislocation nucleation versus cleavage failure ahead of the crack tip. The growth asymmetry was governed by the relative angle of the slip plane to the crack plane $(\theta)$, and the angle between normal to the crack tip and slip direction $(\phi)$, as depicted in Figure 1. The critical energy release rate $(\mathrm{G})$ required for a dislocation emission can be expressed as follows:

$$
G_{d i s l}=8\left(\frac{1+(1-\vartheta) \tan ^{2} \phi}{(1+\cos \theta) \sin ^{2} \theta}\right) \gamma_{u s}
$$

where $\gamma_{u s}$ is the unstable stacking fault energy for the slip plane. Furthermore, the Rice criterion for examining the competition between dislocation nucleation and cleavage behavior (i.e., $\mathrm{G}_{\text {cleav }}$ $=2 \gamma_{s}$ ) ahead of the crack tip is given by

$$
\frac{\gamma_{s}}{\gamma_{u s}}>4\left(\frac{1+(1-\vartheta) \tan ^{2} \phi}{(1+\cos \theta) \sin ^{2} \theta}\right)
$$


where $\gamma_{s}$ is the surface energy required to create a free surface along the GB interface. Wang et al. $[13,15]$ used the Rice condition to examine experimentally-observed directional crack growth behavior in $\mathrm{Fe}-\mathrm{Si}$ and $\mathrm{Cu}$ bicrystalline interfaces and found that the large variation in crack growth behavior was observed when the ratio of $\frac{\gamma_{s}}{\gamma_{u s}}$ was the lowest. For example, using the RT criterion, Wang et al. $[13,15]$ calculated the $G_{\text {disl }}$ for a dislocation nucleation from the $\Sigma 9(221)$ GB in $\mathrm{Cu}$ and $G_{\text {disl }}$ was $\sim 5.5 \mathrm{~J} / \mathrm{m}^{2}$ and $\sim 2.4 \mathrm{~J} / \mathrm{m}^{2}$ along the $-\mathrm{X}$ and $+\mathrm{X}$ directions, respectively. On the other hand, the critical energy for the cleavage $\left(G_{\text {clea }}\right)$ in the $\Sigma 9$ (221) GB interfaces was $\sim 2.5 \mathrm{~J} / \mathrm{m}^{2}$. These predictions for the $\Sigma 9$ (221) GB suggest that the fracture behavior changes from a ductile to brittle or vice versa, when the crack propagation direction along the interface changes, which was further corroborated with experimental findings. However, a complete understanding of the fundamental mechanisms associated with asymmetric deformation are inaccessible with experimental techniques, especially the atomic deformation ahead of an intergranular crack tip, the role of GB character, and the effects of boundary conditions.

Atomic-scale simulations offer advantages for examining the role of interface in fracture behavior, albeit at very small length and time scales. Nonetheless, such simulations can help establish the relationship between microstructure and material properties. There have been a few noteworthy atomistic studies to understand the atomic scale relationship between the GB structure and the directional crack tip responses along the GB interface [20-22] in $\mathrm{Cu}$ and Al. The general consensus of these studies was that the Rice criterion underestimates the critical energy release rate required for the dislocation emission from certain GBs. For example, Cheng et al. [20] observed heterogeneous dislocation emissions from $\Sigma 9(221)$ GB sites at some distance ahead of the crack tip, instead of a dislocation emission from the crack tip as suggested by the Rice criterion. The complexity of some of the GB SU interactions with the crack tip stress fields induced by boundary conditions could clarify such discrepant views (see experimental work of Wang et al. [13,15] and atomistic work of Cheng et al. [20]) and the applicability of the Rice criterion.

In the present work, the directional anisotropy of an intergranular crack (incipient plastic or cleavage event) along with the role of geometry (crack size, shape) and loading conditions were studied for $<110>$ STGBs in Al. The Rice criterion predictions for the directional behavior of incipient plastic/cleavage event ahead of a crack tip in these GBs were compared against the observed crack tip event. An middle-tension $(\mathrm{M}(\mathrm{T})$ ) specimen was modeled using molecular 
dynamics with a constant strain rate applied normal to the GB plane at $300 \mathrm{~K}$. The tensile stressstrain response of these $\mathrm{M}(\mathrm{T})$ specimen GBs was analyzed to correlate the mechanical response to the GB structure. The observed incipient crack tip event deviates from the Rice criterion predictions for certain interfaces (e.g., $\Sigma 9$ (221), $\Sigma 11$ (332), and $\Sigma 33$ (441) GBs).

Furthermore, the effect of boundary conditions was examined to understand the discrepancy between the predicted and observed incipient behavior during the dynamic fracture using $\mathrm{M}(\mathrm{T})$ specimens. The Mode-I crack propagation simulations were performed using molecular statics (MS) with the displacement field corresponding to a semi-infinite crack being subject to stress intensity factor $\left(\mathrm{K}_{\mathrm{I}}\right)$ at the infinity. The findings of Mode-I fracture were evaluated with regards to the incipient inelastic event ahead of the crack tip along both directions of crack propagation. The effect of boundary condition on the crack tip response was especially evident for the $\Sigma 9$ (221) GB that underwent dislocation nucleation from GB sources present at some distance ahead of the crack tip during the constant tensile strain rate loading. On the other hand, for the same GB during the Mode-I fracture simulation; we observed the crack tip behavior consistent with the Rice criterion. These results clarify the discrepant view of experimental observations $[13,15]$ and the modeling work [20], and should put to rest the viability of the Rice criterion. Lastly, the

effects of applied boundary conditions on the incipient crack tip responses were explained with the help of K-dominance zone ahead of a crack tip [23], these findings highlight the importance of employing well-controlled loading conditions during atomistic fracture studies.

\section{Computational Methods}

A parallel molecular dynamics code (large-scale atomic/molecular massively parallel simulator, LAMMPS [24]) with a semi-empirical embedded atom method (EAM) [25] potential was used to study the role of GB character on plastic events and the subsequent crack dynamic behavior in Al STGBs with $<110>$ tilt axis. In this work, we employed the EAM potential of Liu et al. [26] to describe the interactions between Al-Al and for generating the impurity-free GB systems. The EAM potential was fitted to the experimental data and a set of ab-initio structural atomic forces calculated for large set of configurations. Furthermore, this potential has been shown to accurately reproduce the experimental solid-liquid binary phase diagram [27], thus quantifying stable structure formations and thermo-mechanical behavior of nanocrystalline materials [28] and elucidating the mechanistic causes for the crossover from a dislocation to a 
grain boundary-based deformation mechanism with decreasing grain size, grain boundary structure and energies, and many other physical behaviors, including the stacking fault energy (SFE) in Al [27]. The unstable stacking fault energy of aluminum using the EAM potential of Liu et al. [26] is $0.128 \mathrm{~J} / \mathrm{m}^{2}$ at $0 \mathrm{~K}$ [29]. The stable and unstable SFEs are critical intrinsic material properties when modeling extended defects such as dislocations and GBs.

\subsection{Equilibrium grain boundary structures}

The GB structure and minimum energy were calculated using a bicrystal simulation cell with three-dimensional (3D) periodic boundary conditions consisting of two grains at $0 \mathrm{~K}$ as described by Rittner and Seidman [30]. The periodic boundaries were maintained with a separation distance of $12 \mathrm{~nm}$ between the boundaries. The several $0 \mathrm{~K}$ minimum energy GB structures were obtained through successive rigid body translations followed by an atom deletion technique and energy minimization using a non-linear conjugate method [30-37]. In particular, grain boundary structures generated using this technique have been compared with experimental high-resolution transmission electron microscopy images [38-40].

Atomic rearrangement that occurs during minimization of the GB can be expressed as relative displacement of atomic planes normal to the GB [41]. This provides a microscopic measure for the excess volume or the free volume required to create the interface because the strain field associated with the interface at the atomistic scale decays as $e^{-z}$, where $\mathrm{z}$ is the distance normal to the GB plane. The free volume can be measured by finding the relative displacement of two parallel atomic planes nearest to the GB. This measure represents the local expansion undergone by crystals at the interface $\left(\Delta z^{*}\right)$.

\subsection{Molecular dynamic fracture simulation using $M(T)$ specimens}

For dynamic fracture simulations, the minimized GB structures were modeled by prescribing periodic conditions along the $\mathrm{Y}$ and $\mathrm{Z}$ directions and a non-periodic condition along the $\mathrm{X}$ direction (Figure 2a). Prescribing a periodic boundary condition along the $\mathrm{Y}$ direction introduced a second GB interface at the end of the simulation setup, but the influence of this interface on the results was minimized by prescribing sufficient separation between the two GB planes ( $\sim 25 \mathrm{~nm})$. The dimensions along the $\mathrm{X}$ and $\mathrm{Z}$ directions were chosen to provide sufficient atomic layers for convergence of the 3D interfacial fracture. The atomistic model was equilibrated at $300 \mathrm{~K}$ using 
a canonical ensemble (NVT) for $5 \mathrm{~ns}$ and the periodic directions were subsequently relaxed using the isothermal-isobaric (NPT) equations of motions for $10 \mathrm{~ns}$. Then, a through-thickness crack with a $2 \mathrm{~nm}$ height was introduced along the GB plane by deleting atoms within the crack region as illustrated in Figure $2 \mathrm{a}$, such that the $2 a / W$ ratio for the atomistic model was kept constant at 0.1 , where $2 a$ is the crack length, and $W$ is the total width of the specimen (X dimension, 50 $\mathrm{nm})$. The minimum dimensions for the entire bicrystal at equilibrium were approximately $50 \mathrm{~nm}$ $\times 50 \mathrm{~nm} \times 5 \mathrm{~nm}(\sim 1.0$ million atoms $)$. The overall dimensions, crack size, and the crack profile used with the $\mathrm{M}(\mathrm{T})$ geometry is similar to that of what has been reported in literature for various other mechanics studies, such as [3,29,42-44]. The model was deformed under tensile loading by applying a constant strain rate of $10^{8} \mathrm{~s}^{-1}$ perpendicular to the GB plane (Y direction), while the transverse boundaries were controlled using the NVT ensemble equations of motion for a temperature of $300 \mathrm{~K}$. The NVT boundary conditions produce a tensile stress in the transverse direction, which affect the interface strength [45]. However, this lateral NVT boundary condition was deemed more appropriate than a stress-free NPT ensemble, as an NPT boundary condition relaxes the transverse stresses and inhibits crack propagation. The lateral stresses were on the order of $1 / 7$ th to $1 / 10$ th of the applied stress in the $\mathrm{Y}$ direction. Each bicrystal interface was deformed up to an engineering strain of $8 \%$. Note that we also carried out MS simulations for a few GBs using a constant strain rate on $\mathrm{M}(\mathrm{T})$ specimen and observed similar plastic (dislocation nucleation) events as compared to the MD counterpart. Finally, the crack size and the overall geometry of both specimens (Mode-I and $\mathrm{M}(\mathrm{T})$ ) are comparable to what has been used in the open literature [2,3,20,29,42-44,46,47]. We have also systematically compared the effect of crack sizes $(\mathrm{a} / \mathrm{W})$, geometries, different interatomic potentials (see Appendix) and have validated that the behavior observed is consistent with that in the open literature.

\subsection{Molecular statics simulation for Mode-I crack propagation of bicrystals}

Molecular statics fracture simulations (Figure 2b) were performed for few $<110>$ STGBs ( $\Sigma 9$ (221) and $\Sigma 27$ (552)) using the displacement field corresponding to a semi-infinite crack being subject to $K_{I}$ at the infinity. These simulations were used to quantify the critical stress intensity factor for the first plastic/cleavage event from the crack tip and to corroborate with the Rice predictions (Eq. 2). The cylindrical bicrystal specimens were loaded along the $\mathrm{X}(u), \mathrm{Y}(v)$ and Z (w) directions using following conditions: 


$$
\begin{gathered}
u(r, \theta)=K_{I} \frac{1+\vartheta}{E} \sqrt{\frac{r}{2 \pi}} \cos \frac{\theta}{2}\left(2-4 \vartheta+2 \sin ^{2} \theta\right) \\
v(r, \theta)=K_{I} \frac{1+\vartheta}{E} \sqrt{\frac{r}{2 \pi}} \sin \frac{\theta}{2}\left(4-4 \vartheta+2 \cos ^{2} \theta\right) \\
w(r, \theta)=0
\end{gathered}
$$

where $K_{I}$ is the stress intensity factor for a Mode-I loading, $\vartheta$ is the Poisson's ratio (0.3), E is the Young's modulus (75 GPa), and $\mathrm{r}$ and $\theta$ are the polar coordinates defined from the crack tip as shown in Figure 1a. These displacement boundary conditions were applied to atoms within $1 \mathrm{~nm}$ of the cylindrical surface, as shown in Figure 2b. The displacement fields were varied by incrementally changing the stress intensity factor $\left(\Delta \mathrm{K}=1.5 \times 10^{-3} \mathrm{MPa} \sqrt{m}\right)[48,49]$. The boundary along the $\mathrm{Z}$ direction was modeled with a periodic boundary condition, while the $\mathrm{X}$ and $\mathrm{Y}$ boundaries were modeled as free surfaces. The minimum dimension for the entire cylindrical bicrystal at equilibrium was approximately $20 \mathrm{~nm}$ in radius and was $4 \mathrm{~nm}$ thick along the $\mathrm{Z}$ direction ( $\sim 500,000$ atoms). Atoms were deleted to create an atomistically sharp crack of 4 $\mathrm{nm}$ length. Each displacement increment was followed by a nonlinear conjugate gradient energy minimization process.

The virial definition of stress was used to quantify macroscopic responses of the interface [50]. Note that virial stresses can be significantly different from continuum stresses at the free surfaces. The evolution of the defected atoms (e.g., the crack tip, dislocations, and twins) was visualized using the centrosymmetry parameter [51]. This method accurately identifies defects within the crystal structure and is able to separate these defects from regions of large elastic deformation, while retaining the symmetry of their local environment during elastic deformation. The crack tip propagation was tracked using the centrosymmetry parameter; a cutoff of the centrosymmetry parameter $(>20)$ was used to identify surface atoms and track the crack trajectory. 


\section{Results and Discussion}

\subsection{Characteristics of GBs: Structural unit, and GB energy}

Understanding the structure and energy of the GB system is crucial for engineering materials intended for advanced applications because GB properties can vary widely (coherent twin versus low angle versus high angle GBs). In this study, a range of GB structures and energies that are representative of some of the variation observed in the GB character distribution of polycrystalline as well as nanocrystalline metals was used to investigate the role of GB character on plastic events such as dislocation emission, twin formation, and atomic displacements at the interface with a preexisting crack, see Table I.

The SUs associated with each investigated $<110>$ STGB are shown in Figure 4 with the help of Voronoi atomic volume and summarized in Table 1, along with the $0 \mathrm{~K}$ GB energy, the free volume, and the SU periodic length of each interface. The orientation of available in-plane slip systems with respect to the GB plane correlate the amount of free volume (Voronoi atomic volume) available at the interface and degree of atomic rearrangement required for heterogeneous dislocation nucleation along slip planes (Figure 4). The GB SU descriptions are in good agreement with previous descriptions available in literature [52,30,53]. The $\Sigma 3$ (111) coherent twin boundary with the 'D' SU (Shockley partial dislocation) along the GB plane, that exhibited small variation in the Voronoi volume at the interface. The $\Sigma 11$ (113), and $\Sigma 33$ (225) GB's exhibit a smaller excess atomic volume peak at the GB. This is primarily due presence of diamond shaped ' $\mathrm{C}$ ' SU that consists of 4 atoms forming a relatively tightly packed interface. The 'E' SU is made up of 6 atoms arranged in a kite shape. On the other hand, the $\Sigma 27$ (552) GB exhibits a large variation in the atomic volume at the interface, implying that the initial GB interface has a large available excess volume to accommodate deformation. The presence of large pockets of excess volume available in the GBs containing ' $E$ ' SUs at the termination of $\{111\}$ planes explains ease in a nucleating ISF that in turn lead to the dislocation nucleation [54]. These pockets of excess volume along the interface also promote cleavage behavior along the interface in the absence of dislocation nucleation ahead of the crack tip.

\subsection{Molecular dynamics: $M(T)$ specimens}

The dynamic fracture using $\mathrm{M}(\mathrm{T})$ specimens at $300 \mathrm{~K}$ by applying a constant tensile strain rate normal to the GB plane were performed. The stress-strain response and its correlation to the 
GB structures are discussed. This is followed by quantifying directional crack tip responses by quantifying the incipient plastic/cleavage events with applied strains.

\subsubsection{Mechanical responses}

The mechanical response under tension of $<110>$ STGBs as a function of applied normal strain is shown in Figure 4. The mechanical behavior can be grouped into two distinct sets: one set for GBs with a structural period containing the ' $E$ ' $S U$ and another for GBs without the ' $E$ ' SU. The presence of the 'E' SU lowers the dislocation nucleation stress, in agreement with other studies [52,53]. The maximum stress for all of the $<110>$ STGBs evaluated was between 1.62 $\mathrm{GPa}(\Sigma 9(221))$ and $2.70 \mathrm{GPa}(\Sigma 11(113))$, showing the strong effect of the GB structure. Qualitatively, the average maximum normal interface strength for GBs containing the 'E' SU is about $1.63 \mathrm{GPa}$ as compared to other non-'E' SU GBs which is about $2.52 \mathrm{GPa}$. This clearly underlines the influence of GB structural unit on the crack tip deformation, as the similar maximum stress for GBs with the 'E' SU suggest that GB dislocations are driving the incipient plastic activity.

\subsubsection{Analyzing deformation at/ahead of the crack tip}

A detailed analysis of incipient crack tip behavior was performed by visualizing the atomic region along the interface ahead of the mobile crack tip. The centrosymmetry parameter was used to detect dislocations and stacking faults, which have larger centrosymmetry values than atoms within a pristine fcc lattice. The two prominent deformation modes observed in the following study are highlighted below.

\subsubsection{Directional anisotropy: $\Sigma 11(113)$ GB}

The atomic events ahead of the crack tips of $\Sigma 11$ (113) GB during fracture were examined to understand the predicted directional anisotropy (Table 2). At the applied strain of 2.6\% (Figure

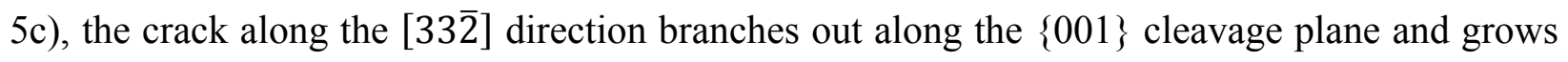
in a brittle manner. On the other hand, along the [3 $\overline{3} 2]$ direction, large amount of atomic movement was witnessed at the interface to nucleate a partial dislocation for crack growth along the $[\overline{3} \overline{3} 2]$ direction (Figure 5e). As listed in Table 2, the relative angle between the preferred slip system and the crack plane of $100^{\circ}$ requires a large amount of energy to nucleate and propagate 
dislocations away from the crack tip. The relative orientation of slip planes with respect to the crack plane and the crack growth direction, was the principal cause for the crack asymmetry observed during the failure of the interface (refer Table 2 and Figure 5).

\subsubsection{Directional anisotropy: $\Sigma 27(552) \mathrm{GB}$}

In grain boundaries with the 'E' SU, directional anisotropy of an intergranular crack during the dynamic fracture was observed. Moreover, the ' $E$ ' structural unit was identified as the cause for the twin deformation. For example, in the case of the $\Sigma 27$ (552) GB, along the [115] direction, a kink was observed in the bottom grain ahead of the crack tip which was attributed to nucleation of a microcrack along the $\{001\}$ plane followed by the nucleation of a partial dislocation and the subsequent absorption at the kink surface, as shown in Figure 6a-b. With an increase in the applied strain to about $2 \%$, nucleation of the leading partial dislocation along (111) $[11 \overline{2}]$ from the ISF was observed, as shown in Figure 6c. The extent of atomic displacement required to nucleate the partial dislocation was negligible; this behavior was found in all the GBs containing the 'E' SU. In an attempt to further clarify the observed deformation behavior with molecular dynamics, we also performed MS simulations using $\mathrm{M}(\mathrm{T})$ specimens and observed similar incipient crack tip event compared to the MD counterpart. Also, the behavior ahead of the crack tip was further validated for a specimen with different crack sizes $(\mathrm{a} / \mathrm{W})$, geometries, different interatomic potentials.

The atomic deformation ahead of the crack tip along the [1]15] direction is shown in Figure $6 \mathrm{~d}$. The nucleation of (111)[11 $\overline{2}]$ leading partial dislocation at $\varepsilon=1.7 \%$ left behind a SF in its wake, as shown in Figure 6d. The analysis of the deformation behavior ahead of the crack tip along both [115] and [ $\overline{1} \overline{1} 5]$ directions shows that relatively minimal applied strain was required to cause the 'E' SU to rearrange and form an ISF along the interface. This ISF acted both as an accelerant in the plastic events at the interface and as a source for nucleating partial dislocations. Also, the discrete nature of these plastic events were significantly affected by the combination of slip planes orientation with respect to the crack, the free volume, and SU notably not just the SU or the GB energy alone.

In summary, the observed incipient crack tip plastic event (Table 3) was compared against theoretically predicted behavior (Table 2). The comparison clearly shows that the observed behavior was different from the predicted behavior during dyamic fracture of the GB interface. 
However, the observed plastic events at the crack tip for the $\Sigma 11$ (113) GB are in agreement with previous MD studies [20] and experimental work [13]. The majority of GBs show dislocation nucleation from GB sources at a distance ahead of the crack tip (Table 3), which raises questions about the applicability of the Rice criterion. Note that Cheng and co-workers [20,21] have observed a similar dislocation emission in the case of 'E' SU GBs with a sharp edge crack, i.e., dislocation nucleation occurred from GB sources ahead of the crack tip instead of the crack tip. Furthermore, we validated this behavior using various crack sizes and geometries. Thus, it is plausible that the incipient plastic events observed in our work are largely influenced by boundary conditions instead of the crack geometry examined here.

\subsection{Mode-I crack propagation}

The effect of boundary conditions on crack tip plasticity was examined to understand the discrepancy between the theoretical predictions and MD observations. Molecular statics simulations for Mode-I crack propagations were carried out by applying displacements in accordance to the $\mathrm{K}_{\mathrm{I}}$ solution at a semi-infinite crack tip for the $\Sigma 9$ (221) and $\Sigma 27$ (552) STGBs. At the point of instability, i.e., due to a dislocation emission or a cleavage-based crack extension, we measured the critical stress intensity factor $\left(\mathrm{K}_{\text {critical }}\right)$. The incipient crack tip event was then compared against theoretical predictions and the critical energy release rates $\left(\mathrm{G}_{\text {critical }}\right)$ were quantified. Furthermore, intergranular crack tip responses computed using Mode-I boundary conditions were compared with the MD simulation responses. In the next subsection, the nucleation of plastic events and the directional crack tip response along the GB for the (a) $\Sigma 9$ (221) and (b) $\Sigma 27$ (552) STGBs, which contain a kite shape 'E' SU, are compared with the theoretical predictions using the Rice criterion.

\subsubsection{Mode-I crack propagation: $\Sigma 9$ (221) STGB}

Dynamic fracture simulations of the $\Sigma 9$ (221) GB exhibits multiple dislocation emissions, which was observed from GB sites at some distance $(\sim 10-20 \AA)$ ahead of the crack tip. This raises questions about the applicability of the Rice criterion, which suggest a dislocation emission from the crack tip. To further explore this observed behavior, the effect of boundary conditions on the crack tip plasticity was examined. In the case of the $\Sigma 9$ (221) STGB, the available slip systems along the $+X[11 \overline{4}]$ direction are at $15.8^{\circ}$ and $125.3^{\circ}$ with respect to the 
crack plane (221), as shown in Figure 7a. The Rice criterion predicts a critical energy release rate of $3.62 \mathrm{~J} / \mathrm{m}^{2}$ for the dislocation nucleation along the $[11 \overline{4}]$ direction. However, the energy release rate required for the GB cleavage is about $0.92 \mathrm{~J} / \mathrm{m}^{2}$ (refer Table 2). Thus, predicting a cleavage behavior ahead of the crack tip along the $[11 \overline{4}]$ direction. The observed initial activity along the $[11 \overline{4}]$ direction was the nucleation of a partial dislocation at a very low energy release of 0.19 $\mathrm{J} / \mathrm{m}^{2}$ (Figure $7 \mathrm{~b}$ ). The partial dislocation was unable to propagate through the grain giving rise to subsequent cleavage fracture along the $\{001\}$ planes within the grains, as shown in Figure 8c. The $\{001\}$ free surface formed within the grain further dissociates into a dislocation which propagates through the grain (refer Figure 7c). Similarly, along the $-X[\overline{1} \overline{1} 4]$ direction, the angle between feasible slip planes and the crack plane $\{221\}$ are $54.7^{\circ}$ and $164.2^{\circ}$, as shown in Figure $7 \mathrm{a}$, and the cleavage behavior was predicted for the crack tip along this direction (Table 2). Here, we observed nucleation of a partial dislocation ahead of the crack tip with an energy release rate of $0.16 \mathrm{~J} / \mathrm{m}^{2}$ (Figure $7 \mathrm{~d}$ ), followed by another partial dislocation nucleation in the bottom grain (Figure 7e). The measured energy release rate for the dislocation nucleation along the [1̄14] direction was significantly lower than the predicted energy release rate $\left(0.97 \mathrm{~J} / \mathrm{m}^{2}\right)$. This result clearly highlights the importance of the GB dislocation structure on crack tip plasticity, which cannot be predicted accurately by the Rice criterion.

The stress field ahead of the crack tip is important for understanding the directional responses for certain GBs. Hence, it is informative to compare the crack tip plasticity mechanisms from the two types of simulations explored herein: dynamic fracture and Mode-I loading. During the dynamic fracture simulations of the $\Sigma 9$ (221) GB, dislocation nucleation was observed from GB sites at some distance $(\sim 10-20 \AA)$ ahead of the crack tip which also compare well with the works of Cheng et al. [20]. In contrast, during the Mode-I simulations of the $\Sigma 9$ (221) GB, the crack propagation process involved dislocation nucleation directly at the crack tip. This is directly associated with the stress field at the crack tip. Since dislocation nucleation from 'E' SU GB sources requires lower stresses $(<50 \%)$ compared to other $<110>$ GBs $[52,54]$, it is necessary to apply the correct stress field ahead of the crack tip while studying crack directional anisotropy of GB interfaces.

\subsubsection{Mode-I crack propagation: $\Sigma 27$ (552) STGB}

The effect of boundary conditions on the directional anisotropy in the $\Sigma 27$ (552) GB was reexamined under Mode-I conditions. The two possible slip systems along the $+X$ 
[115] direction are at $19.4^{\circ}$ and $129^{\circ}$ with respect to the crack plane (552), as shown in Figure 8a. On the other hand, along the $-\mathrm{X}[\overline{1} \overline{15}]$ direction, the possible slip planes are oriented at an angle of $51^{\circ}$ and $159.6^{\circ}$ with respect to the crack plane (552). The observed behavior for the crack tip

along the $[11 \overline{5}]$ direction under Mode-I boundary conditions was a cleavage fracture along the $\{001\}$ plane ahead of the crack tip (Figure 8b), which is consistent with the predicted incipient plastic event using the Rice criterion at the crack tip (Table 2). The cleavage event was closely followed by the nucleation of partial dislocation from GB sources ahead of the crack tip followed by absorption of the dislocation at the branched crack tip (Figure 8b). As the applied load was

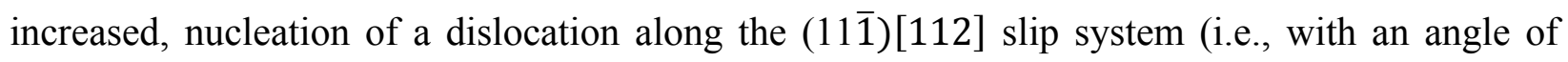
$19.4^{\circ}$ ) from the branched crack tip was observed (Figure 8c). Likewise, for the crack tip along the [115] direction, nucleation of a dislocation with energy release rate of $\mathrm{G}_{n u c l}=0.52 \mathrm{~J} / \mathrm{m}^{2}$ was observed (Figure 8d-e). This energy release rate for dislocation emission was significantly lower than that of the predicted energy release rate (i.e., $\mathrm{G}_{d i s l}=1.04 \mathrm{~J} / \mathrm{m}^{2}$, Table 2). One plausible explanation for this discrepancy is that the presence of the ' $E$ ' SU lowers the activation energy required for nucleation of an ISF.

\subsection{Effect of crack geometry on the crack tip deformation}

The observed incipient crack tip behavior for $\mathrm{M}(\mathrm{T})$ specimens with different crack tip geometries were examined for the $\Sigma 9$ (221) GB. The initial crack was created with an a/W ratio of 0.1 . In order to alter the crack geometry, an extra periodic length of atoms were removed along the GB to generate a sharper crack tip for the M(T) specimen (Figure 9), the crack height was varied (5 $\AA$ and $10 \AA$ ) for the $\Sigma 9$ (221) GB (Figure 10). The simulations were carried out at $0 \mathrm{~K}$ and loading conditions were similar to those described earlier. For the crack height of 0.5

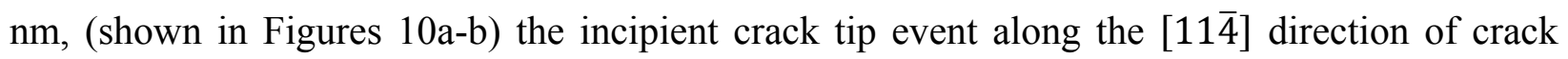
propagation takes places from GB sources at a distance of $1 \mathrm{~nm}$ ahead of the crack tip (Figure 10a). A large number of partial dislocations were nucleated from GB sources (Figure 10b). Similar behavior was observed for the $\mathrm{M}(\mathrm{T})$ specimen with crack height of $1 \mathrm{~nm}$ (Figures 10c-d). In contrast, during the Mode-I simulations of the $\Sigma 9$ (221) GB, the crack propagation process involved dislocation nucleation directly at the crack tip consistent with the Rice criterion. The behavior ahead of the crack tip was further examined for a specimen with a longer crack $(\mathrm{a} / \mathrm{W}=$ 0.3 ) in the $\Sigma 9$ (221) GB (Figure 11). Here, the crack height was $1 \mathrm{~nm}$ and the crack was created 
using the same technique described earlier, i.e., an extra periodic length of atoms were removed along the GB to generate a sharper crack profile. For the larger crack size $(\mathrm{a} / \mathrm{w}=0.3)$, we observed similar incipient plastic events as was in the case of $\mathrm{a} / \mathrm{w}=0.1$. These findings suggest that, despite the crack geometry, the GB sites ahead of the crack tip still remain preferable sites for the dislocation nucleation. In literature, the effect of crack size/geometry on the competition between cleavage and dislocation nucleation is well established [55].

To understand the insensitivity of incipient activities towards variations in crack sizes/crack geometries for an $\mathrm{M}(\mathrm{T})$ specimen, we explored the notion of K-dominance region ahead of the crack tip. The K-dominance zone is defined as the region where the singular crack tip stress contributes $95 \%$ of the total crack tip stresses (singular and non-singular). In cases, when the singular stress field at the crack tip is not large enough to contain the entire fracture process, then the non-singular stress near the crack tip can influence the fracture mechanism [23]. In other words, as the crack size becomes smaller, the K-dominance region decreases as well and this leads to underestimation of the stress intensity factor for the incipient crack tip events [20,21]. Thus, the study on the effect of crack geometry on incipient crack tip event discussed here suggests that the entire fracture process may not be contained in the fracture zone for the case of $\mathrm{M}(\mathrm{T})$ specimen; hence, the $\mathrm{M}(\mathrm{T})$ specimen $\mathrm{K}$-dominance zone size is insufficient to study incipient crack tip events at the nanoscale. Finally, the application of $\mathrm{K}_{\mathrm{IC}}$ as a fracture criterion to study nanoscale fracture needs to be carried out by selecting appropriate loading conditions and geometry to contain the entire fracture zone.

In summary, MD simulations with certain GBs, such as $\Sigma 3$ (111), $\Sigma 9$ (221), $\Sigma 33$ (441) and $\Sigma 11$ (332) GB, show dislocation emission from a GB source at a finite distance $(\sim 1 \mathrm{~nm})$ ahead of the crack tip rather than from the crack tip, as suggested by the Rice criterion. However, these observations are consistent with previous findings (see Cheng et al. [20,21]). In an attempt to understand discrepancy between the theoretical predictions and atomistic observations, the effect of boundary conditions ( $\mathrm{M}(\mathrm{T})$, Mode-I and the edge crack) on the crack tip events was examined and it was concluded that the incipient plastic events observed were strongly influenced by the boundary conditions (i.e. activation of dislocation sources along the GB, in contrast to dislocation nucleation directly from the crack tip. The influence of the boundary conditions on the crack tip response was especially notable for the $\Sigma 9$ (221) GB. For example, the Mode-I fracture simulation show crack tip behavior consistent with the Rice criterion. These 
results highlight the importance of boundary/loading conditions on the nanoscale investigation of fracture. These results clarify the discrepant view of experimental observations $[13,15]$ and the modeling work [20], and outline the underlying shortcomings of fracture specimen that were not subjected to far-field $\mathrm{K}_{\mathrm{I}}$ displacement corresponding to an semi-infinite crack.

\section{Conclusions}

In this work, the directional anisotropy of an intergranular crack was studied for $<110>$ STGBs in Al using atomistic simulations. Of particular interest was how the GB character affects the incipient crack tip event, i.e. competition between dislocation nucleation and cleavage fracture and its comparison with the Rice criterion. Following conclusions can be drawn from the present study:

1. The normal stress response for the $<110>$ GBs during dynamic fracture, we observed two distinct responses $\left(\theta<109.47^{\circ}\right.$ and $\left.\theta>109.47^{\circ}\right)$ of interface strength and the GB misorientation. This clearly underlines the influence of GB structural unit on the crack tip deformation, as the GBs with the ' $\mathrm{E}$ ' SU have similar maximum strength suggesting that the GB dislocations were driving the incipient plastic activity ahead of the crack tip.

2. With $\mathrm{M}(\mathrm{T})$ specimens, dislocation nucleation from GB sites for some GBs ( $\Sigma 9$ (221), $\Sigma 11$ (332) and $\Sigma 33$ (441)) occurred at some distance ahead of the crack tip, in agreement with the findings of Cheng et al. [20] (with shaper edge crack specimens). The observed incipient crack tip plasticity for different crack sizes with these GBs question the applicability of the Rice criterion for predicting behavior of all interfaces.

3. In an attempt to understand the discrepancy between the theoretical predictions and MD/MS observations with an $\mathrm{M}(\mathrm{T})$ specimen for incipient crack tip behavior, the effect of boundary conditions on the crack tip events was examined. The incipient plastic events were strongly influenced by the stress state ahead of the crack tip (i.e. activation of dislocation sources along the $\mathrm{GB}$, in contrast to dislocation nucleation directly from the crack tip). The $\mathrm{M}(\mathrm{T})$ specimen's K-dominance zone size is insufficient to study incipient crack tip events at the nanoscale. These findings outline shortcomings of a nanoscale fracture specimen not subject to a far-field $\mathrm{K}_{\mathrm{I}}$ displacement corresponding to a semi-infinite crack. 
4. Last, the GB structural unit plays a large part in dislocation nucleation ahead of the crack tip, as interface atoms provide varying degrees of mobility to incorporate plastic deformations. These new atomistic findings provide a physical basis for recognizing the role of the GB character and the boundary conditions on intergranular crack behavior.

\section{Acknowledgement}

The authors would like to recognize Dr. W. Mullins and Dr. A.K. Vasudevan from the Office of Naval Research for providing insights and valuable suggestions. This material is based upon work supported by the Office of Naval Research under contract no. N000141110793. We would also like to acknowledge the Fulton High Performance Computing at Arizona State University and the anonymous reviewers for their helpful comments. 


\section{Appendix}

\section{A1. Effect of loading conditions}

In addition to the Mode- $\mathrm{I}\left(\mathrm{K}_{\mathrm{I}}\right)$ and $\mathrm{M}(\mathrm{T})$ specimen findings described in the result section, we have also examined the incipient plastic event with an edge crack specimen containing the $\Sigma 9$ (221) GB. The edge crack specimen was created with a crack length of $4 \mathrm{~nm}$ and the crack mouth displacement of $2 \mathrm{~nm}$ with the crack tip along the [11 $\overline{4}]$ direction at the GB. The sides (along the $\mathrm{X}$ direction) of the specimen were prescribed to a zero displacement boundary condition in the $\mathrm{X}$ and $\mathrm{Z}$ directions, while the GB was pulled under uniaxial tension with strain rate of $10^{8} \mathrm{~s}^{-1}$ (along the Y direction). Subsequently, energy minimization was carried out at $0 \mathrm{~K}$ for the mobile atoms in the specimen. The incipient crack tip event along the $[11 \overline{4}]$ direction of crack growth was emission of partial dislocation from GB sources located about $10 \AA$ ahead of the crack tip (Figure A1c). These findings they are in agreement with the previously discussed results for $\mathrm{M}(\mathrm{T})$ specimen and similar results were reported by Cheng et al [20]. These results again highlight the importance of the loading conditions on the incipient crack tip behavior (Figure A1).

\section{A2. EAM potential validation}

In an attempt to be certain that these findings were not an artifact of the chosen EAM potential [26], we simulated the $\mathrm{M}(\mathrm{T})$ specimen with crack height of $1 \mathrm{~nm}$, using the Mishin et al [56] EAM potential for Al. During the deformation of the $\Sigma 9$ (221) GB, the partial dislocation were nucleated from GB sources ahead of the crack tip that formed a twin on further increasing applied strain (Figure A2). 


\section{References}

[1] Yip S, Wolf D. Atomistic Concepts for Simulation of Grain Boundary Fracture, in: Mater. Sci. Forum, vol. 46. 1991.

[2] Farkas D, Duranduru M, Curtin WA, Ribbens C. Philos Mag A 2001;81:1241.

[3] Hai S, Tadmor E. Acta Mater 2003;51:117.

[4] Li H, Chandra N. Int J Plast 2003;19:849.

[5] Zhu T, Li J, Yip S. Phys Rev Lett 2004;93:025503.

[6] Zhu YT, Liao XZ, Srinivasan SG, Zhao YH, Baskes MI, Zhou F, Lavernia EJ. Appl Phys Lett 2004;85:5049.

[7] Zhang ZJ, Paulino GH. Int J Plast 2005;21:1195.

[8] Buehler MJ, Gao H. Nature 2006;439:307.

[9] Yamakov V, Saether E, Phillips DR, Glaessgen EH. J Mater Sci 2007;42:1466.

[10] Yamakov VI, Glaessgen EH. Nat Mater 2007;6:795.

[11] Bobylev SV, Mukherjee AK, Ovid'ko IA, Sheinerman AG. Int J Plast 2010;26:1629.

[12] Solanki KN, Ward DK, Bammann DJ. Metall Mater Trans A 2011;42:340.

[13] Wang J-S, Anderson PM. Acta Metall Mater 1991;39:779.

[14] Saeedvafa M. Mech Mater 1992;13:295.

[15] Wang J-S, Mesarovic SD. Acta Metall Mater 1995;43:3837.

[16] Kysar JW. Acta Mater 2000;48:3509.

[17] Kysar JW. J Mech Phys Solids 2001;49:1099.

[18] Tang H, Acharya A, Saigal S. Mech Mater 2005;37:593.

[19] Rice JR. J Mech Phys Solids 1992;40:239.

[20] Cheng Y, Jin Z-H, Zhang YW, Gao H. Acta Mater 2010;58:2293.

[21] Cheng Y, Shi MX, Zhang YW. Int J Solids Struct 2012;49:3345.

[22] Glaessgen EH, Phillips D, Saether E, Yamakov V. 2006.

[23] Sun CT, Qian H. J Mech Mater Struct 2009;4:743.

[24] Plimpton S. J Comput Phys 1995;117:1.

[25] Daw MS, Baskes MI. Phys Rev B 1984;29:6443.

[26] Liu XY, Ercolessi F, Adams JB. Model Simul Mater Sci Eng 2004;12:665.

[27] Buehler MJ. Atomistic Modeling of Materials Failure. Springer; 2008.

[28] Wolf D, Yamakov V, Phillpot SR, Mukherjee A, Gleiter H. Acta Mater 2005;53:1.

[29] Tadmor E, Hai S. J Mech Phys Solids 2003;51:765.

[30] Rittner J, Seidman D. Phys Rev B 1996;54:6999.

[31] Rhodes NR, Tschopp MA, Solanki KN. Model Simul Mater Sci Eng 2013;21:035009.

[32] Tschopp MA, McDowell DL. Philos Mag 2007;87:3871.

[33] Tschopp MA, McDowell DL. Int J Plast 2008;24:191.

[34] Solanki KN, Tschopp MA, Bhatia MA, Rhodes NR. Metall Mater Trans A 2013;44:1365.

[35] Bhatia MA, Solanki KN. J Appl Phys 2013;114:244309.

[36] Rajagopalan M, Tschopp MA, Solanki KN. JOM 2014;66:129.

[37] Rajagopalan M, Bhatia MA, Tschopp MA, Srolovitz DJ, Solanki KN. Acta Mater 2014;73:312.

[38] Mills MJ, Daw MS, Thomas GJ, Cosandey F. Ultramicroscopy 1992;40:247.

[39] Medlin DL, Mills MJ, Stobbs WM, Daw MS, Cosandey F. HRTEM Observations of a $\Sigma=3$ $\{112\}$ Bicrystal Boundary in Aluminum, in: MRS Proc., vol. 295. 1992. 
[40] Grigoriadis P, Karakostas T, Komninou P, Pontikis V. Low-Energy Configurations of the $\Sigma=5$ (210)[001] Tilt Grain Boundary in FCC Crystals, in: Mater. Sci. Forum, vol. 294. 1998.

[41] Chen SP, Srolovitz DJ, Voter AF. J Mater Res 1989;4:62.

[42] Song J, Curtin WA. Nat Mater 2012.

[43] Xu GQ, Demkowicz MJ. Phys Rev Lett 2013;111:145501.

[44] Zhang J, Ghosh S. J Mech Phys Solids 2013;61:1670.

[45] Kitamura T, Yashiro K, Ohtani R. JSME Int J Ser A 1997;40:430.

[46] Yamakov V, Saether E, Phillips DR, Glaessgen EH. Phys Rev Lett 2005;95:15502.

[47] Warner D, Curtin W, Qu S. Nat Mater 2007;6:876.

[48] Ringdalen Vatne I, Stukowski A, Thaulow C, Østby E, Marian J. Mater Sci Eng A 2013;560:306.

[49] Ko W-S, Jeon JB, Lee C-H, Lee J-K, Lee B-J. Model Simul Mater Sci Eng 2013;21:025012.

[50] Tsai DH. J Chem Phys 1979;70:1375.

[51] Kelchner CL, Plimpton SJ, Hamilton JC. Phys Rev B 1998;58:11085.

[52] Spearot DE, Jacob KI, McDowell DL. Int J Plast 2007;23:143.

[53] Sansoz F, Molinari JF. Acta Mater 2005;53:1931.

[54] Tschopp MA, Tucker GJ, McDowell DL. Acta Mater 2007;55:3959.

[55] Beltz GE, Lipkin DM, Fischer LL. Phys Rev Lett 1999;82:4468.

[56] Mishin Y, Farkas D, Mehl MJ, Papaconstantopoulos DA. Phys Rev B 1999;59:3393. 


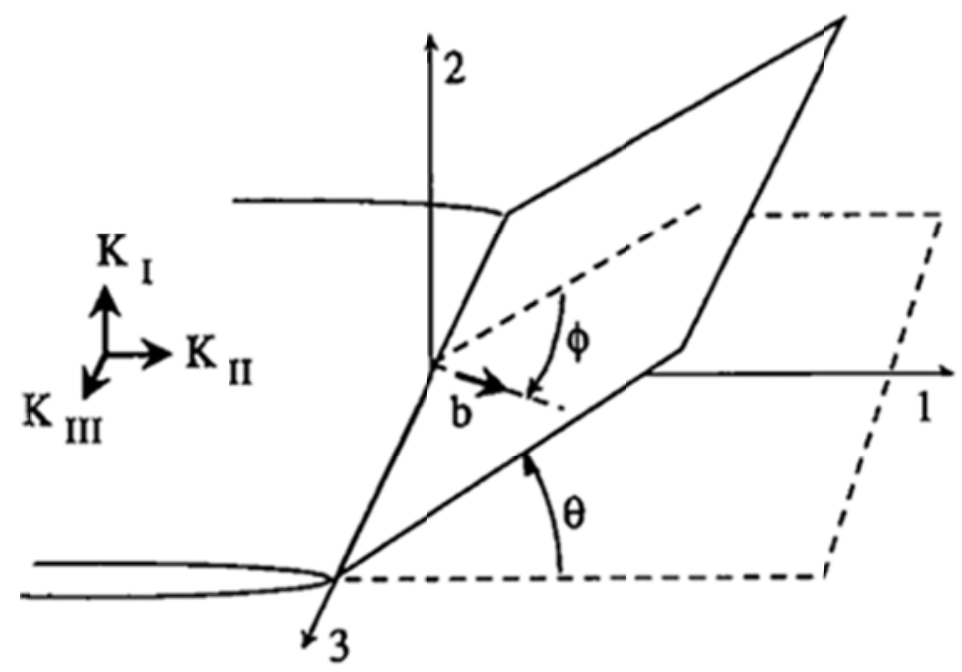

Figure 1. A schematic representation of geometric variables $\theta$ and $\phi$ in the Rice criterion, adapted from [19]. The slip plane inclined at an angle $\theta$, and the crack plane and slip direction inclined at an angle $\phi$ with the crack growth direction.

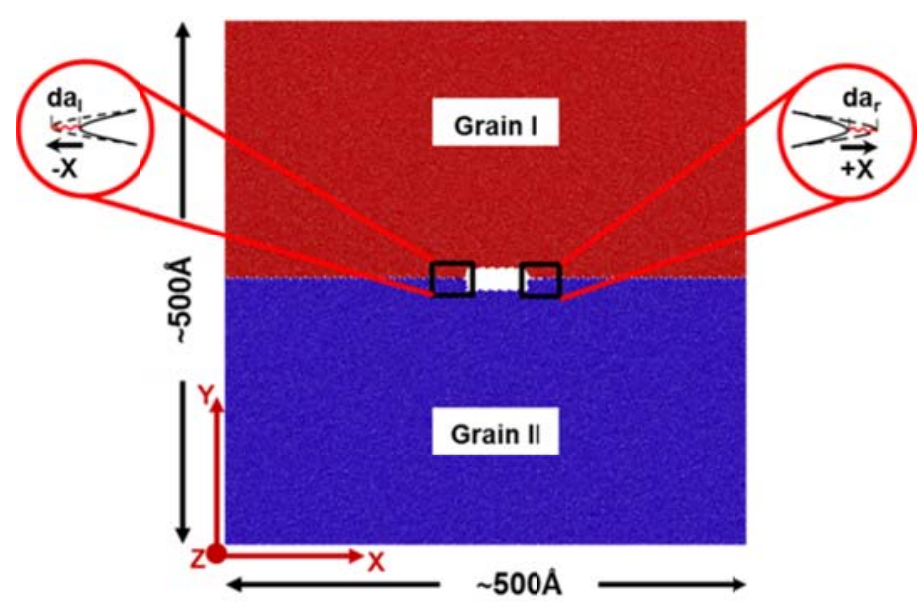

a)

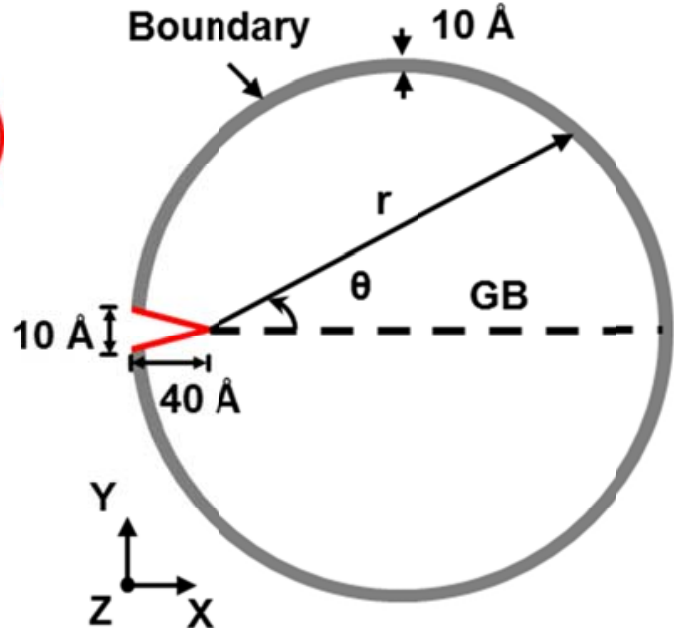

b)

Figure 2. a) Atomistic cell with a GB interface perpendicular to the $Y$ axis. The red and blue regions are grains I and II, respectively. For grains I and II, the $\mathrm{Z}$ tilt axis is the same for both orientations, i.e., $<100>$ or $\langle 110>$. Tensile load was applied along the $\mathrm{Y}$ axis to investigate GB SUs' role on plastic events and any subsequent interfacial failure behavior. b) The geometric representation of fracture specimen where the Mode-I loading was applied by displacing boundary atoms using MS framework. 


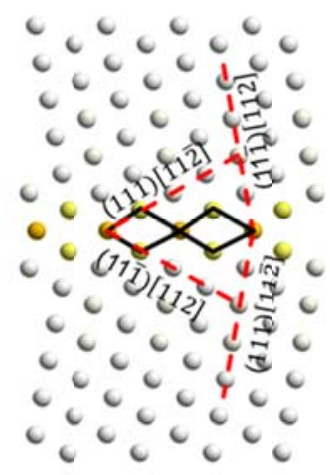

(a) $\Sigma 11(113) 50.48^{\circ}$ |C.C|

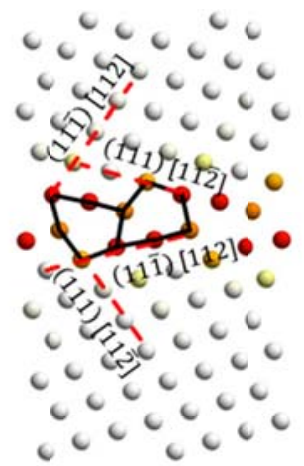

(e) $\Sigma 9$ (221) $141.06^{\circ}$

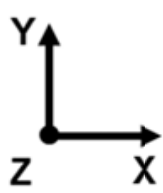

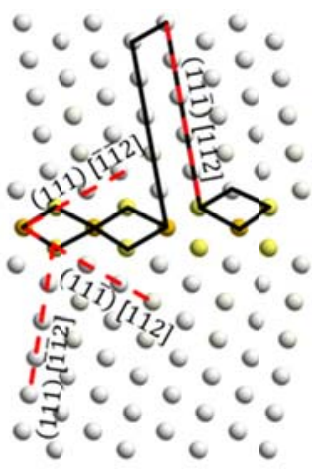

(b) $\Sigma 33(225) 59^{\circ}$ |C.CDC.C|

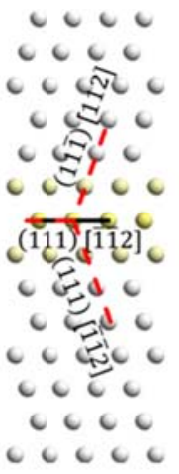

(c) $\Sigma 3$ (111) $109.48^{\circ}$

|D.D|

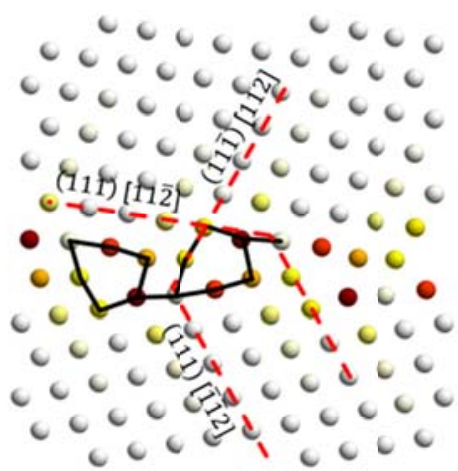

(d) $\Sigma 11(332) 129.52^{\circ}$

|DE.DE|

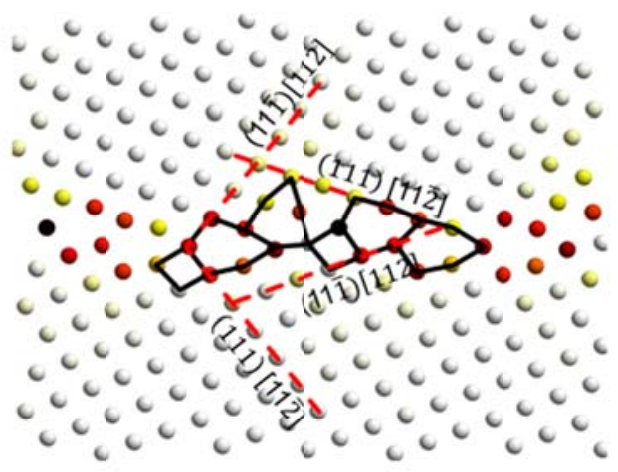

(f) $\Sigma 27$ (552) $148.42^{\circ}$ |AEE.AEE|

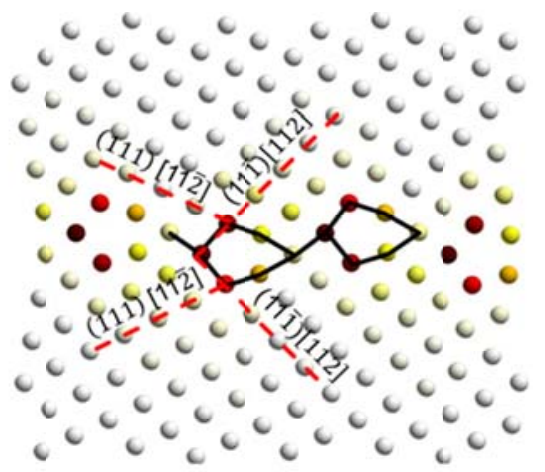

(g) $\Sigma 33$ (441) $159.96^{\circ}$ |ED.ED|

\section{$V_{\text {voro }}$}

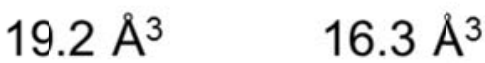

Figure 3. $<110>$ symmetric tilt GB structures with structural units outlined for a) $\Sigma 11$ (113) $\left(\theta=50.48^{\circ}\right)$, b) $\Sigma 33(225)\left(\theta=59^{\circ}\right)$, c) $\Sigma 3(111)\left(\theta=109.47^{\circ}\right)$, d) $\Sigma 11$ (332) $\left(\theta=129.52^{\circ}\right)$, e) $\Sigma 9$ (221) $\left(\theta=141.06^{\circ}\right)$, f) $\Sigma 27(552)\left(\theta=148.52^{\circ}\right)$ and g) $\Sigma 33(441)\left(\theta=159.96^{\circ}\right)$. Atoms are colored based on Voronoi volume on a scale of $19.2 \AA^{3}$ to $16.3 \AA^{3}$. The favorable slip planes (111) and (111) are denoted using dotted lines. This helps in visualizing the initial orientation of slip planes with respect to the GB structure and the amount of excess volume available to the GB atoms in order to nucleate a dislocation along these slip planes. 


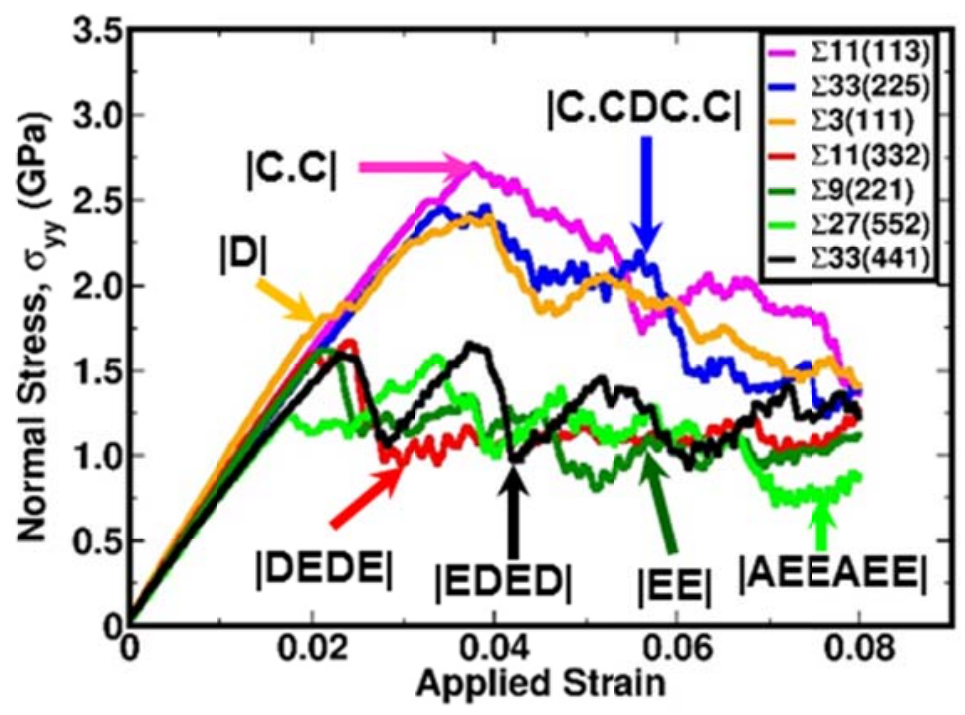

Figure 4. Stress-strain behavior of various $<110\rangle$ GB interfaces under tensile loading. The simulation results indicate influence of SU on the maximum normal stress encountered by the GB interface, especially the 'E' SU. 

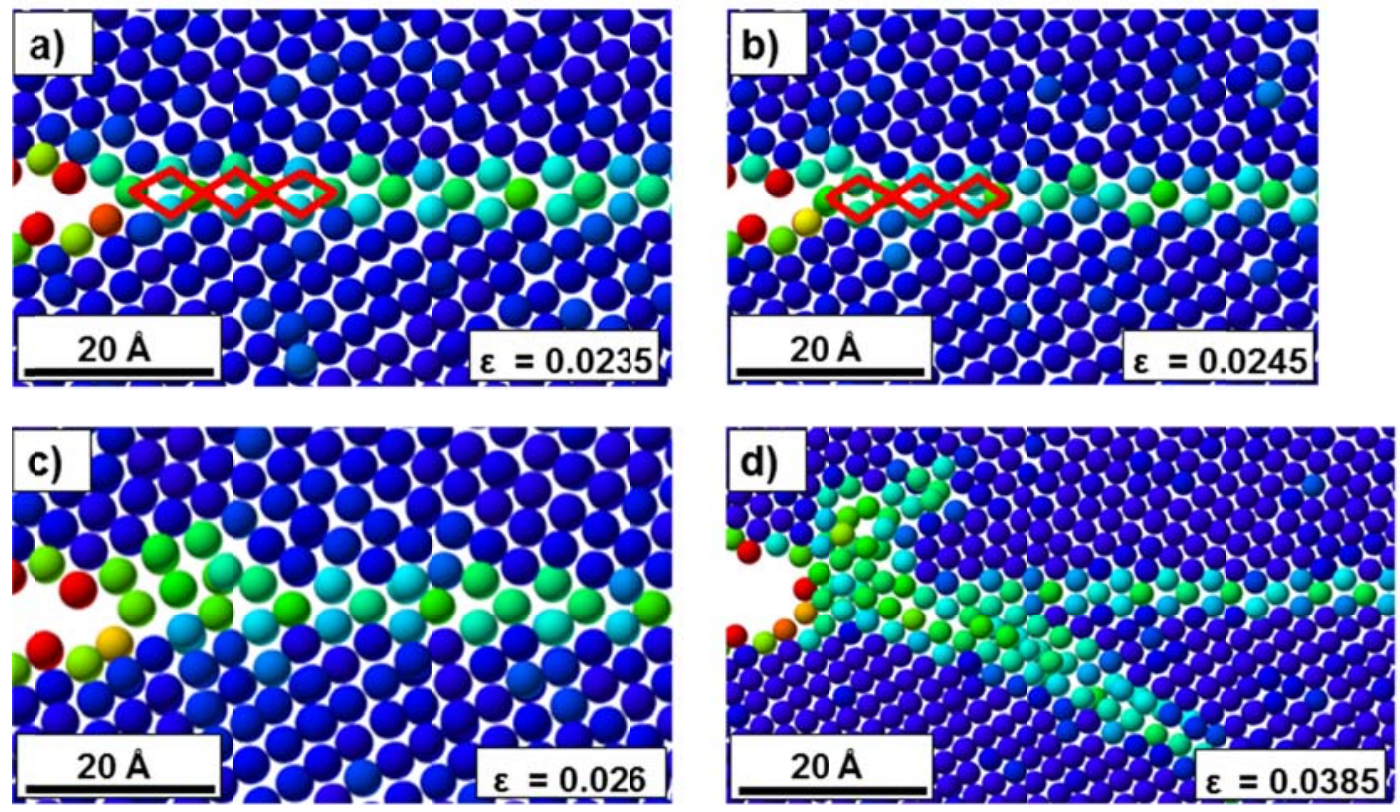

csym
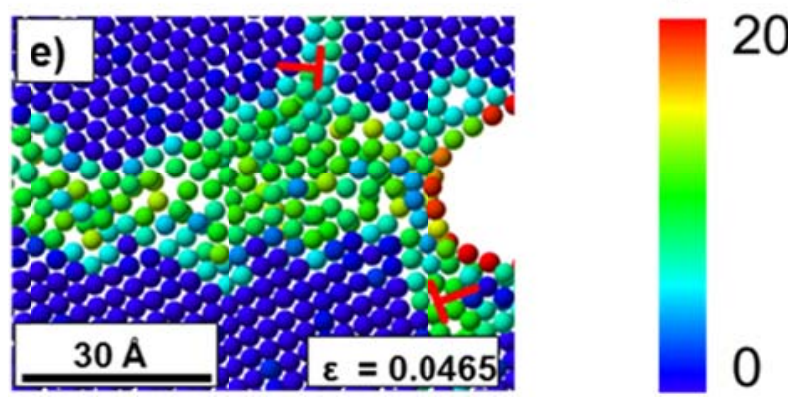

Figure 5. The plastic events across the $\Sigma 11$ (113) GB interface with atoms were identified using the centrosymmetry parameter. (a-d) show plastic events during the crack growth along the

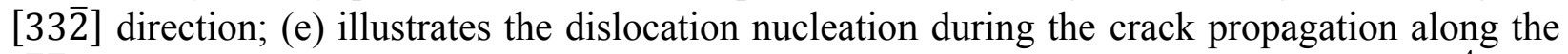
[3 $\overline{3} 2]$ direction. Note that the equivalent applied strain for an elapsed time of $1 \mathrm{ps}$ is $10^{-4}$ (i.e., the applied strain rate of $10^{8}$ ). 

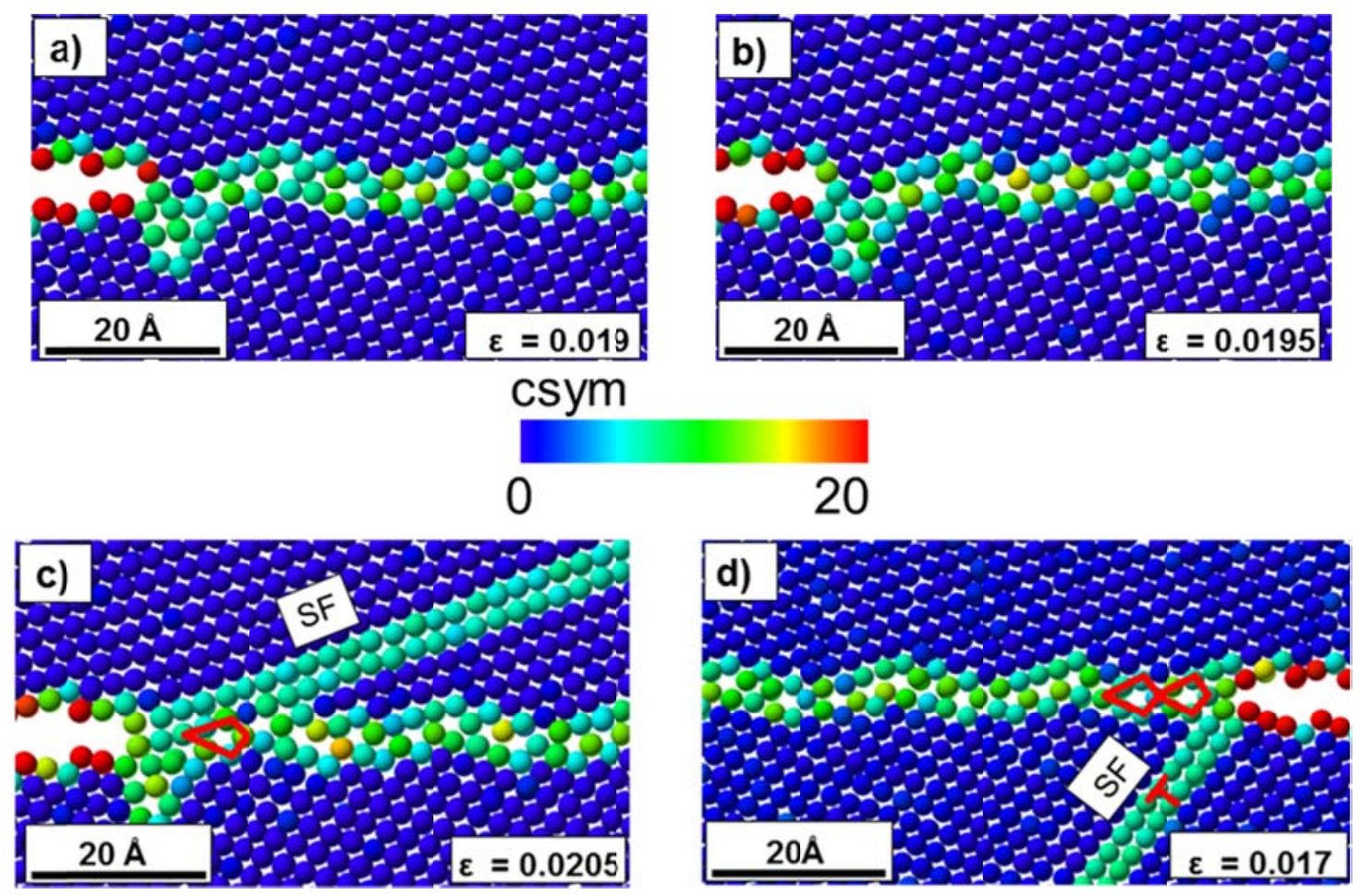

Figure 6. The plastic events across the $\Sigma 27$ (552) GB interface with atoms are identified using centrosymmetry. (a-c) Shows a nucleation of partial dislocation along the $[11 \overline{5}]$ direction, and (d) Incipient plastic event at the crack tip along the [115] direction. The equivalent applied strain for an elapsed time of $1 \mathrm{ps}$ is $10^{-4}$ (i.e. applied strain rate of $10^{8}$ ). 

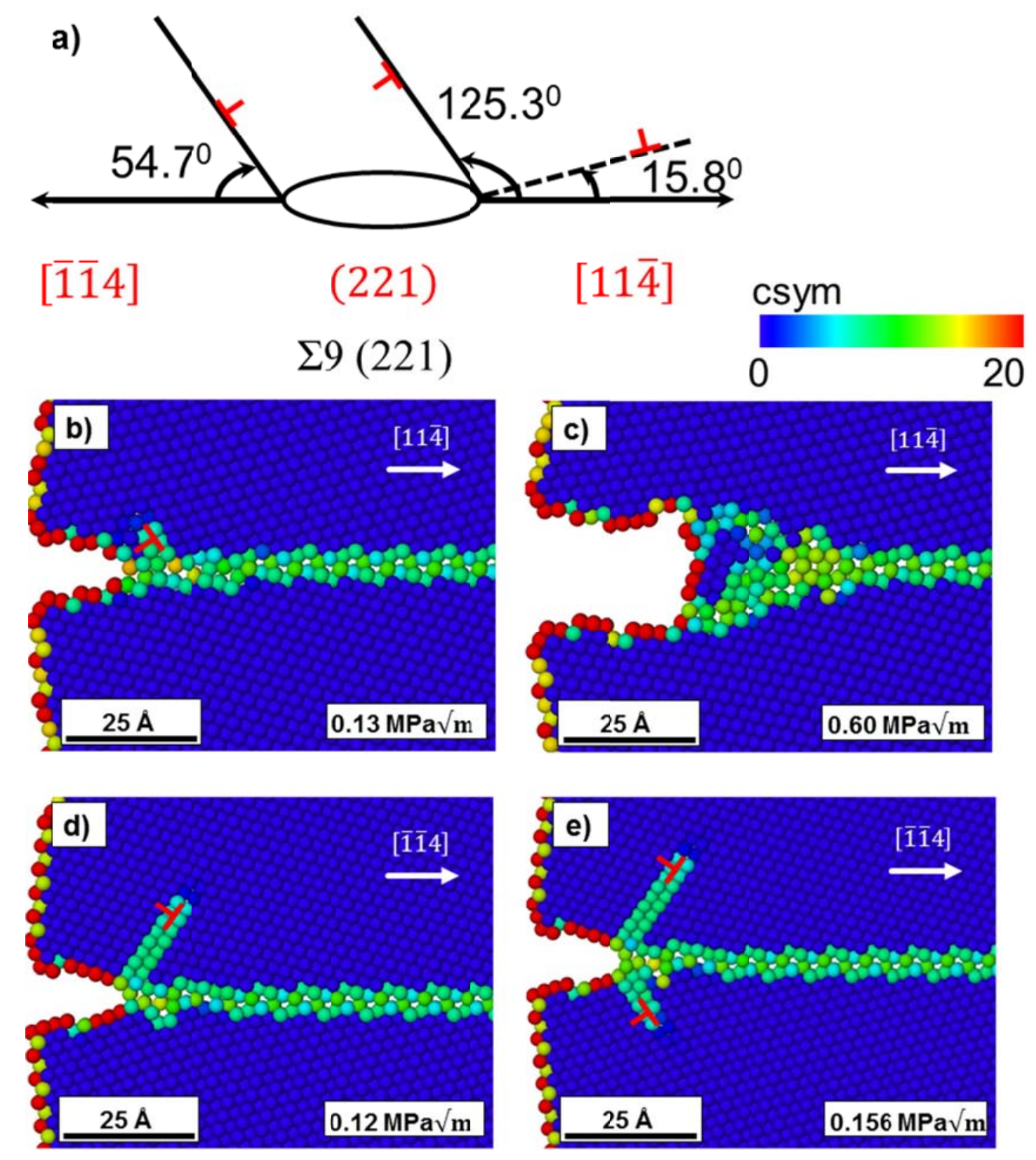

Figure 7. a) An illustration of the preferred slip system orientation with respect to the GB plane for the $\Sigma 9$ (221) GB during crack propagation along both directions. The dashed line depicts the orientation of the second favored slip system. b) Along the [11 $\overline{4}]$ direction of crack propagation, dislocation nucleation takes place along the slip plane oriented at $125.3^{\circ}$. c) The dislocation was unable to propagate away from the crack tip and crack growth changed to cleavage mechanism. d) Along the [1]4] the crack nucleated dislocation, e) with increasing in the stress intensity at the crack tip another dislocation was nucleated in the bottom grain. 

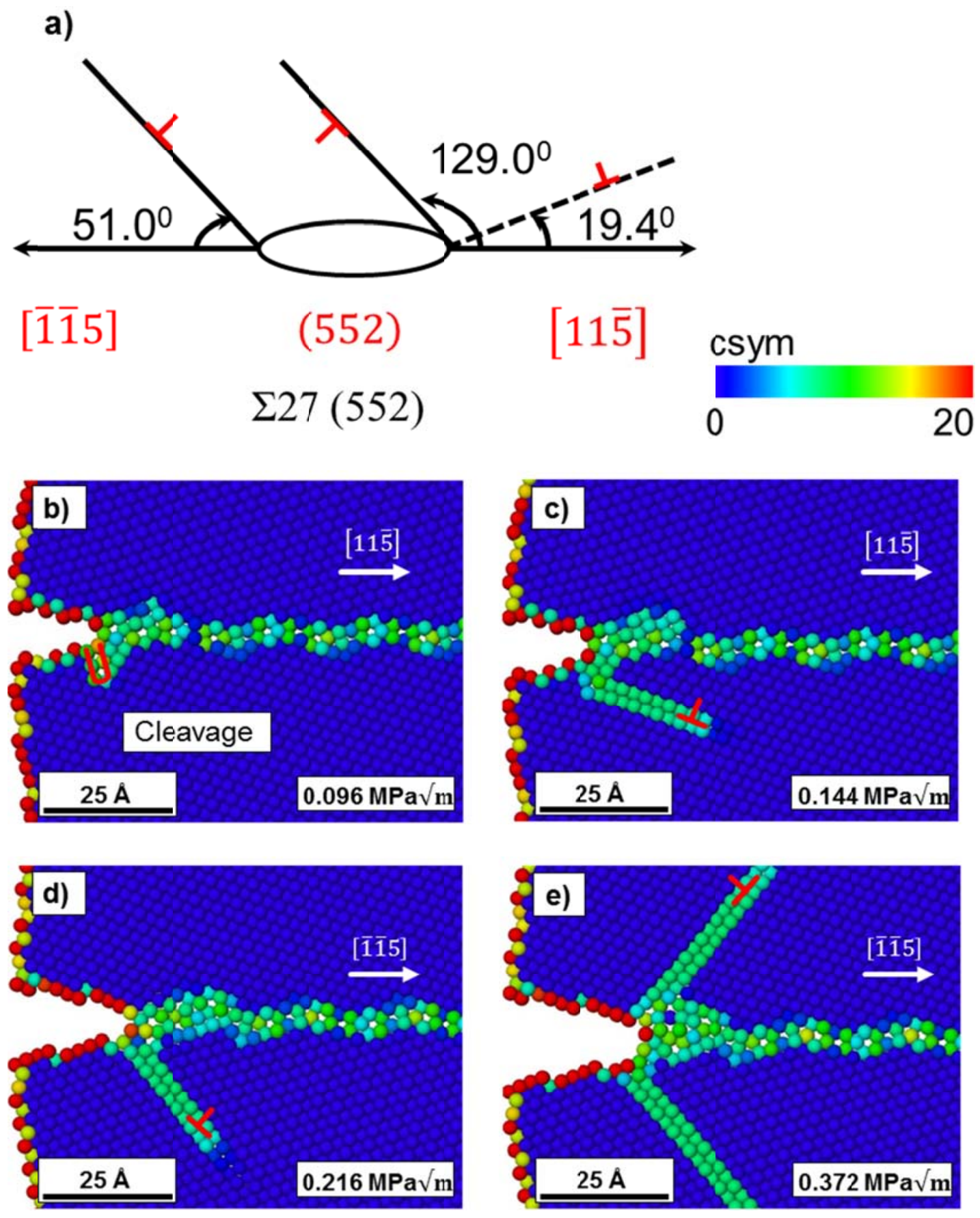

Figure 8. a) An illustration of the preferred slip system orientation with respect to the GB plane for $\Sigma 27$ (552) GB during crack propagation along both directions. The dashed line depicts the orientation of the second favored slip system. b) Along the [115] direction the crack observed cleavage along $\{001\}$ plane, this was followed closely by emission of dislocation from the GB. c) The interaction of cleaved $\{001\}$ surface and the dislocation with increased load generates a dislocation. d) Along the [1]15] the crack nucleated dislocation, e) as the stress intensity factor at the crack tip was increased another dislocation was nucleated in the bottom grain. 


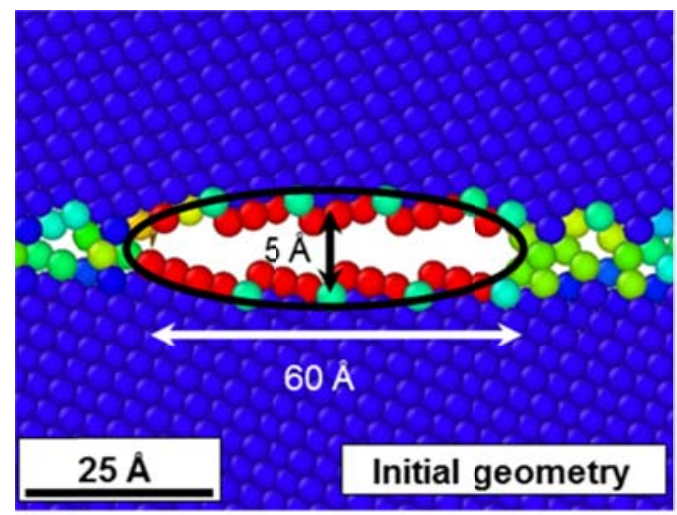

Figure 9. The $\Sigma 9$ (221) GB was evaluated for different crack geometries. The crack heights of 5 $\AA$ and $10 \AA$ were studied. An extra row of atoms along the GB periodic length were deleted to create a sharp crack tip.

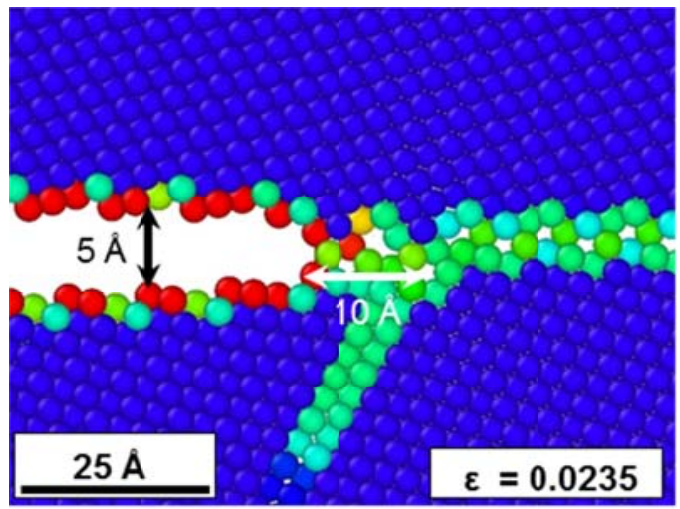

a)

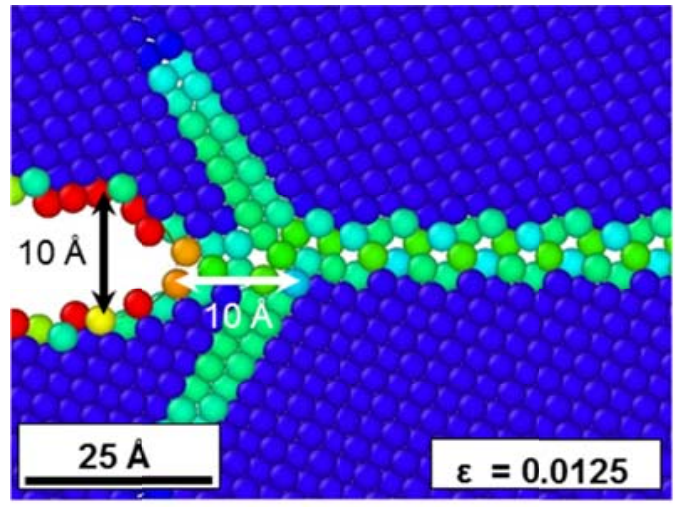

c)

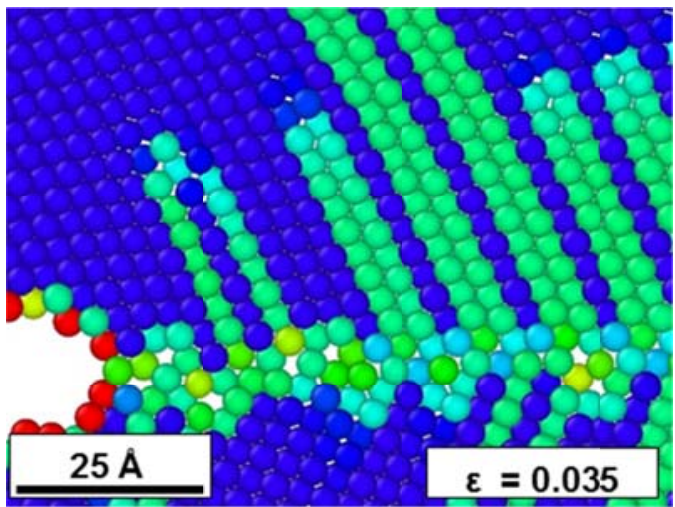

b)

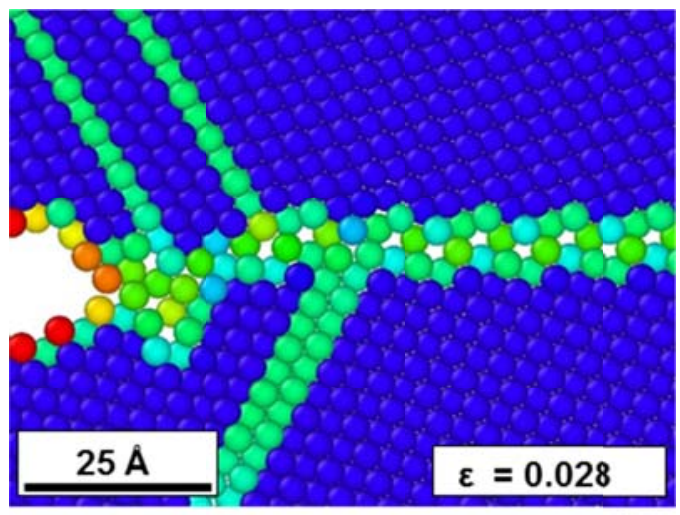

d)

Figure 10. The plastic events ahead of the $\Sigma 9$ (221) GB crack tip model as an M(T) specimen with different crack geometries, i.e., the crack height of $5 \AA$ (a-b) and $10 \AA$ (c-d). In either case, the dislocation nucleation took place about $10 \AA$ ahead of the crack tip and with increasing applied strain lead to the onset of twinning. 


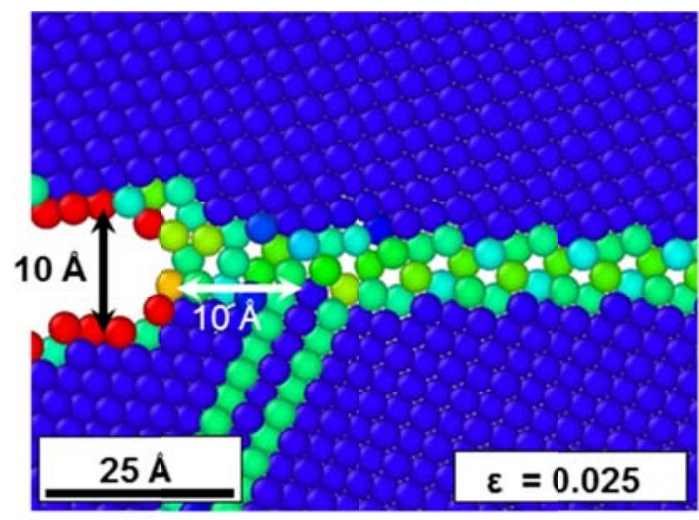

a)

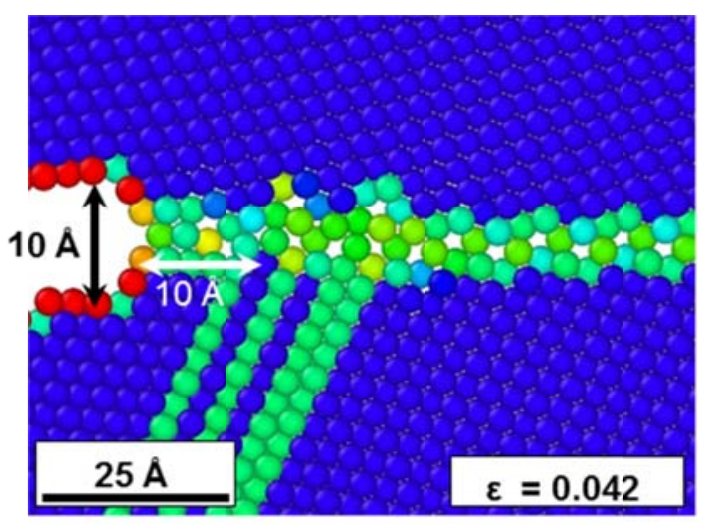

b)

Figure 11. The plastic events ahead of the $\Sigma 9$ (221) GB crack tip model as an M(T) specimen with the crack height of $10 \AA$ and a/W ratio of 0.3 . The incipient plastic events ahead of the crack tip was found to be in agreement with previous results for the crack size of $\mathrm{a} / \mathrm{W}=0.1$. 
Table 1. Grain boundary CSL description, SU notation, the GB periodic length, GB energy, the free volume used in this work. The $\Sigma 27$ (552) GB has the highest initial free volume, periodic length and GB energy among $<110>$ STGBs examined here.

\begin{tabular}{cccccc}
\hline $\begin{array}{c}\text { Misorientation } \\
\text { angle } \\
(\boldsymbol{\theta})\end{array}$ & $\begin{array}{c}\text { CSL } \\
\text { designation } \\
(\boldsymbol{\Sigma})\end{array}$ & $\begin{array}{c}\text { SUM } \\
\text { notation }\end{array}$ & $\begin{array}{c}\text { GB periodic } \\
\text { length }(\mathbf{\AA})\end{array}$ & $\begin{array}{c}\text { GB } \\
\text { energy } \\
\left(\mathbf{m J} / \mathbf{m}^{\mathbf{2}}\right)\end{array}$ & $\begin{array}{c}\text { Free volume } \\
\left(\mathbf{\Delta \mathbf { z } ^ { * } )}\right. \\
\text { in units of } \mathbf{a}_{\mathbf{0}}\end{array}$ \\
\hline $\mathbf{5 0 . 4 8}^{\circ}$ & $\Sigma 11(113)$ & $\mid$ C.C & 18.91 & 151 & 0.1811 \\
$\mathbf{5 9 . 0 0}^{\circ}$ & $\Sigma 33(225)$ & $\mid$ C.CDC.C $\mid$ & 32.75 & 326 & 0.2060 \\
${\mathbf{1 0 9 . 4 7 ^ { \circ }}}^{\circ}$ & $\Sigma 3(111)$ & $\mid$ D.D $\mid$ & 9.87 & 73 & 0.0005 \\
$\mathbf{1 2 9 . 5 2}^{\circ}$ & $\Sigma 11(332)$ & $\mid$ DE.DE $\mid$ & 26.75 & 390 & 0.1724 \\
$\mathbf{1 4 1 . 0 6}^{\circ}$ & $\Sigma 9(221)$ & $\mid$ E.E $\mid$ & 17.10 & 437 & 0.1500 \\
$\mathbf{1 4 8 . 4 2}^{\circ}$ & $\Sigma 27(552)$ & $\mid$ AEE.AEE $\mid$ & 41.90 & 474 & 0.2650 \\
$\mathbf{1 5 9 . 9 6}^{\circ}$ & $\Sigma 33(441)$ & $\mid$ ED.ED $\mid$ & 32.75 & 428 & 0.1610 \\
\hline
\end{tabular}

Table 2. Predicted minimum energy slip plane orientation with respect to the crack plane along both $-\mathrm{X}$ and $+\mathrm{X}$ direction of crack growth $(\theta)$ based on the Rice model (refer equation 1 ). The energy release rate for GB cleavage $\left(\mathrm{G}_{c l e a}=2 \gamma_{s}-\gamma_{g b}\right)$ and dislocation nucleation ahead of the crack tip along both directions.

\begin{tabular}{|c|c|c|c|c|c|}
\hline $\begin{array}{c}\text { Grain } \\
\text { boundary, } \\
\text { CSL } \\
\text { designation } \\
(\Sigma)\end{array}$ & $\begin{array}{c}\text { Critical } \\
\text { energy } \\
\text { release rate } \\
\text { for cleavage } \\
\left(G_{\text {clea }}\right), \mathrm{J} / \mathrm{m}^{2}\end{array}$ & $\begin{array}{l}\text { Relative angle } \\
\text { between slip } \\
\text { plane and } \\
\text { crack plane } \\
\text { along the }-X \\
\text { direction }\left(\theta^{-}\right), \\
\text {degrees }\end{array}$ & $\begin{array}{c}\text { Relative angle } \\
\text { between slip } \\
\text { plane and crack } \\
\text { plane along the } \\
+\mathrm{X} \text { direction }\left(\theta^{+}\right), \\
\text {degrees }\end{array}$ & $\begin{array}{c}\text { Critical energy } \\
\text { release rate for } \\
\text { dislocation } \\
\text { nucleation } \\
\text { along the }-\mathrm{X} \\
\text { direction } \\
\left(G_{\text {disl }}^{-}\right), \mathrm{J} / \mathbf{m}^{2} \\
\end{array}$ & $\begin{array}{c}\text { Critical energy } \\
\text { release rate for } \\
\text { dislocation } \\
\text { nucleation } \\
\text { along the }+\mathrm{X} \\
\text { direction } \\
\left(G_{\text {disl }}^{+}\right), \mathrm{J} / \mathbf{m}^{2} \\
\end{array}$ \\
\hline$\Sigma 11(113)$ & 1.28 & 100.0 & 80.0 & 1.28 & 0.90 \\
\hline$\Sigma 33(225)$ & 1.14 & 95.7 & 84.23 & 1.15 & 0.94 \\
\hline$\Sigma 3$ (111) & 1.23 & 109.5 & 70.5 & 1.73 & 0.86 \\
\hline$\Sigma 11(332)$ & 0.93 & 60.5 & 119.5 & 0.90 & 2.66 \\
\hline$\Sigma 9(221)$ & 0.92 & 54.7 & 125.3 & 0.97 & 3.62 \\
\hline$\Sigma 27(552)$ & 1.28 & 51.0 & 129.0 & 1.04 & 4.56 \\
\hline$\Sigma 33(441)$ & 1.11 & 45.3 & 25.2 & 1.19 & 2.96 \\
\hline
\end{tabular}


Table 3. The observed crack tip events along both directions of the crack propagation for various GBs during the dynamic fracture are summarized. The dislocation (D), dislocation nucleation from GB sites ahead of the crack tip (H.D), and the cleavage (C) are the incipient crack tip events classified below.

\begin{tabular}{|c|c|c|}
\hline $\begin{array}{l}\text { Grain boundary, } \\
\text { CSL designation ( } \Sigma)\end{array}$ & $\begin{array}{l}\text { Incipient plastic event } \\
\text { ahead of the crack tip } \\
\text { along the }-X \text { direction }\end{array}$ & $\begin{array}{l}\text { Incipient plastic event } \\
\text { ahead of the crack tip } \\
\text { along the }+X \text { direction }\end{array}$ \\
\hline$\Sigma 11(113)$ & $\mathrm{D}$ & $\mathrm{C}$ \\
\hline$\Sigma 33(225)$ & $\mathrm{D}$ & $\mathrm{C}$ \\
\hline$\Sigma 3(111)$ & H.D & H.D \\
\hline$\Sigma 11(332)$ & H.D & H.D \\
\hline$\Sigma 9(221)$ & H.D & H.D \\
\hline$\Sigma 27(552)$ & $\mathrm{D}$ & $\mathrm{D}$ \\
\hline$\Sigma 33(441)$ & H.D & H.D \\
\hline
\end{tabular}




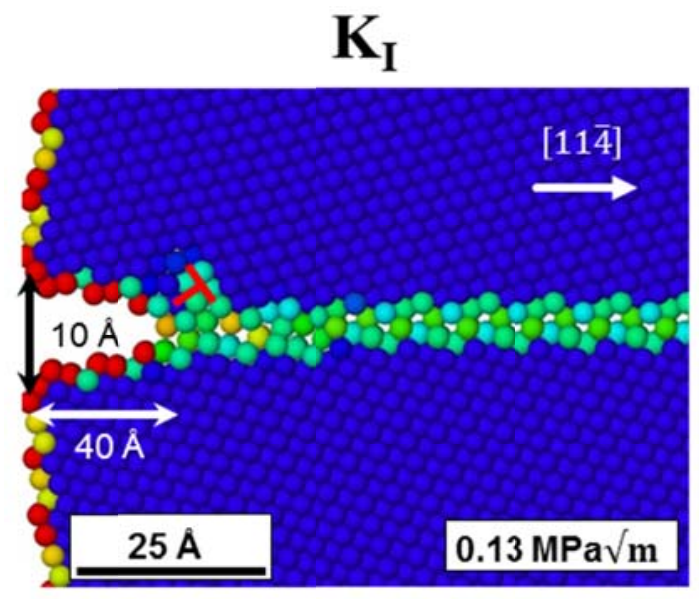

a)

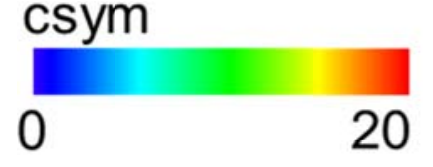

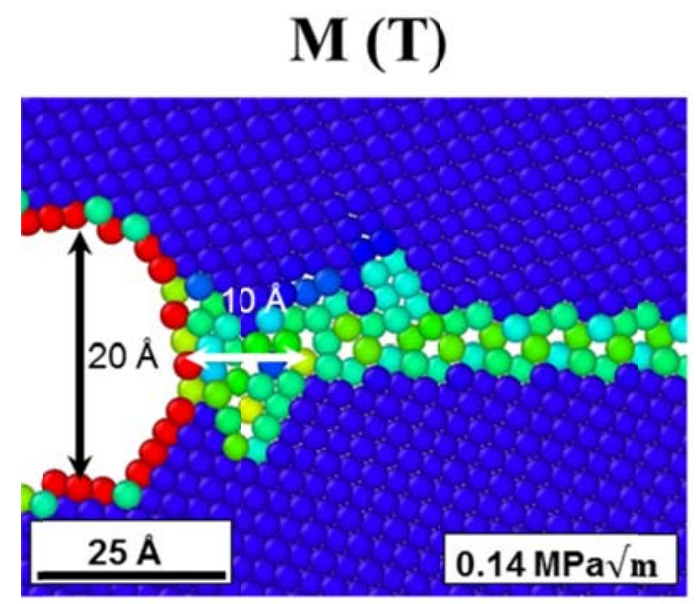

b)

\section{Edge crack}

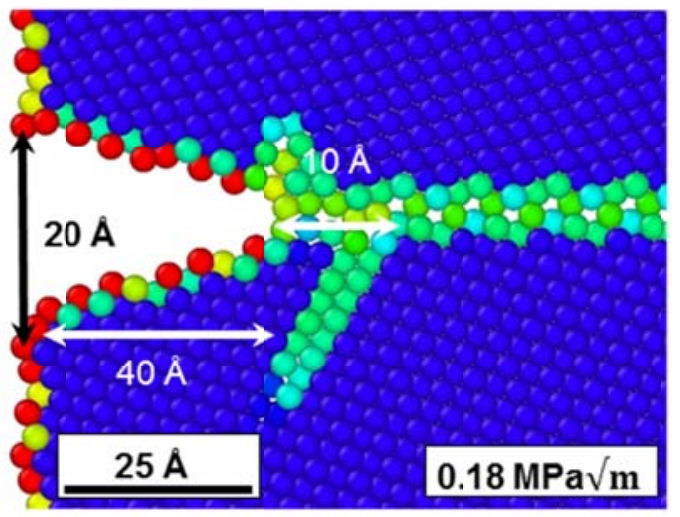

c)

Figure A1. The $\Sigma 9$ (221) GB was evaluated for different loading conditions: a) fracture specimen with the Mode-I loading; b) $\mathrm{M}(\mathrm{T})$ specimen under the Mode-I showing the emission of dislocation at $\sim 10 \AA$ ahead of the crack tip; and c) edge crack specimen under the Mode-I loading with the dislocation emission at $\sim 10 \AA$ ahead of the crack tip. 


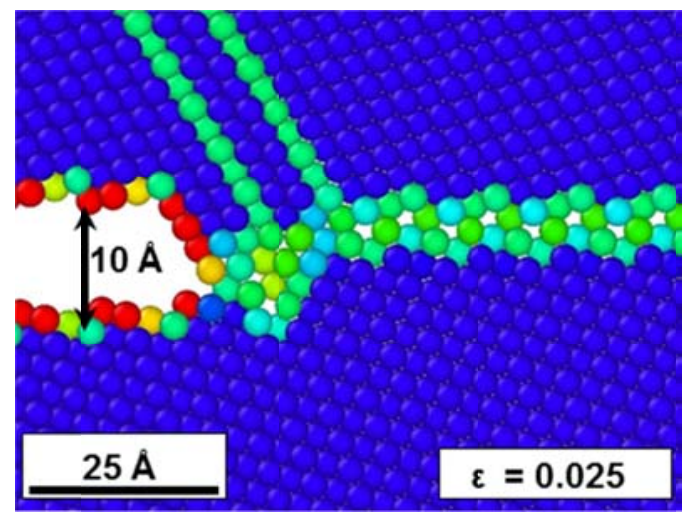

Figure A2. The plastic events ahead of the $\Sigma 9$ (221) GB crack tip model as an M(T) specimen with the crack height of $10 \AA$. Here, we used an Mishin et al [56] EAM potential for Al and the incipient plastic events ahead of the crack tip were found to be in agreement with the Liu et al. [26] EAM potential. 